\title{
Health of Human and Livestock Conceived by Assisted Reproduction
}

\author{
Manon Ceelen and Jan P.W. Vermeiden \\ Research Institute Endocrinology, Reproduction and Metabolism, VU University Medical Centre,The Netherlands
}

\begin{abstract}
$\triangle$ ssisted reproduction is used to resolve infertility problems in human and in breeding programs to generate livestock. Except for gestation length and birth weight, perinatal outcome of children conceived by In Vitro Fertilization is similar to that of spontaneously conceived children. However, large offspring syndrome observed after In Vitro Production in livestock is quite alarming. The distinct parts of assisted reproduction (oocyte maturation, fertilization and culture) have been found to contribute to abnormal fetal growth and development. Genomic imprinting is suggested to be involved in the induction of the aberrant phenotypes observed after assisted reproduction. Furthermore, current knowledge on postnatal health of offspring conceived by assisted reproduction and speculations on potential longterm effects of In Vitro Fertilization will be described.
\end{abstract}

\section{Human Reproduction}

Application of In Vitro Fertilization (IVF) used to overcome reproductive problems in human has rapidly increased since its introduction in 1978. A 1995 collaborative report showed that worldwide each year more than 40000 babies are born after IVF (De Mouzon \& Lancaster, 1997). In 1997, approximately $1 \%$ of all births in the Netherlands have been established by IVF (Buitendijk, 1999).

Each phase of the IVF procedure, including stimulation of multiple folliculogenesis, the process of oocyte retrieval and spermatozoa preparation, fertilization in vitro, culture of the embryo in medium for 48-120 hours and embryo transfer into the uterus instead of the oviduct, is substantially different from natural conception and consequently might have tremendous effects on the developing conceptus (Buitendijk, 1999). The safety of IVF has been evaluated by examination of the course and outcome of IVF pregnancies. Singleton and twin pregnancies following IVF compared with control singleton or twin pregnancies appear to be at increased risk for adverse outcomes, such as preterm birth, perinatal mortality and low birth weight (Koudstaal, et al., 2000; Koudstaal, et al., 2000; Lambalk \& Hooff, 2001). Koudstaal (1999) demonstrated that on average IVF children are 90 gram less in birth weight compared with naturally conceived children. Until now, no indications are present that the incidence of congenital malformations in IVF babies is different from that in normally conceived controls. In summary, only slight differences in perinatal outcome between IVF children and their naturally conceived counterparts have been observed.

\section{Animal Reproduction}

The fear of harmful effects of assisted reproduction on offspring is not totally inequitable as demonstrated by the shortterm effects of animal reproduction. During the last decade, assisted reproductive technologies related to the traditional IVF procedure in human have proven to be a powerful tool in livestock breeding programs (Lohuis, 1995). Most frequently used animal reproduction procedure, called In Vitro Production (IVP), involves the ultrasound guided transvaginal recovery of immature cumulus-oocyte-complexes from ovarian follicles. Following in vitro maturation of collected oocytes, preimplantation embryos are generated in vitro. Main advantages of IVP over traditional superovulation and embryo recovery are increasing genetic variation, reducing generation intervals in females and decreasing breeding risks.

A considerable number of calves and lambs have been born following transfer of IVP embryos. Most offspring appear to be normal, but certain abnormal features have been reported, known as large offspring syndrome (LOS). One of the characteristics of the syndrome is dramatic overgrowth. A small but world-wide inventory combined with data of well designed field studies demonstrated that more than $30 \%$ of the IVP calves, irrespective of their breed, weighed over $50 \mathrm{~kg}$, while birth weight of normally conceived calves is approximately $45 \mathrm{~kg}$ (Kruip \& den Daas, 1997). In addition, IVP sheep embryos resulted in lambs with $10 \%$ higher birth weights compared to in vivo produced sheep embryos (Holm et al., 1996). Other features known to be associated with LOS include increased abortion rate (Hasler et al., 1995), prolonged gestation, delivery problems, increased perinatal mortality, congenital malformations, hydro-allantois (Wagtendonk-de Leeuw et al., 1998) and polyhydramnios (Hasler et al., 1995; Kruip \& den Daas, 1997), enlarged organs (Sinclair et al., 1998) and reluctance to suckle. Described characteristics of LOS are found with varying incidence and severity, indicating that IVP offspring are not equally affected (Young et al., 1998).

Address for correspondence: Manon Ceelen, IVF Centre, Division of Reproductive Endocrinology and Fertility, Research Institute Endocrinology, Reproduction and Metabolism, VU University Medical Centre, de Boelelaan 1117, 1081 HV Amsterdam, The Netherlands. Email:m.ceelen@vumc.nl 
Contrary to LOS in livestock, fetal growth of mice conceived by assisted reproduction is rather retarded. On day 15 of pregnancy, a weight reduction of $30 \%$ was found in mouse fetuses generated by assisted reproduction as compared to their naturally conceived counterparts (Lane \& Gardner, 1997).

\section{Interventions Associated with Aberrant Phenotypes}

Although the direction of abnormal birth weight differs between several mammalian species, it is obvious that fetal growth of offspring generated by assisted reproduction can be affected. It has been speculated that the increased risk in IVF children for adverse perinatal outcome, such as low birth weight, is caused by the risk profile of the mother (Buitendijk, 1999). On average, IVF mothers are older, have had a longer period of attempting to become pregnant, and are more often primiparous than women in the general population. Conversely, in animal reproduction neither the animals donating the gametes nor the recipients suffer from infertility. Therefore, assisted reproduction per se is suggested to contribute to the induction of adverse outcomes in produced offspring. It appeared extremely difficult to determine the contribution of the distinct parts of assisted reproduction, known as oocyte maturation, fertilization and culture, to the observed aberrant phenotypes in offspring. Hormonal stimulation of ovarian activity influences implantation and fetal development in the mouse as demonstrated by Ertzeid and Storeng (2001). A negative effect of ovarian hyperstimulation on oocyte and/or embryo developmental competence was observed as transfer of mice embryos from superovulated donors resulted in a significantly lower implantation rate in control recipients compared with that of embryos from control donors. In addition, transfer of mice embryos recovered from superovulated donors resulted in significantly higher post-implantation fetal mortality in superovulated recipients than in control recipients and the mean weight of live fetuses was significantly lower for fetuses obtained from superovulated recipients compared with that of fetuses obtained from control recipients (Ertzeid \& Storeng, 2001). Therefore, ovarian hyperstimulation in mice can perturb growth and development of the conceptus.

Since ovarian hyperstimulation is a standard part of the IVF procedure, several studies have been published which describe correlations between ovarian hyperstimulation versus reduced birth weight and preterm birth in human. Hyperstimulation has been found to cause low birth weight and increased insulin-like growth factor binding protein levels (Johnson et al., 1995). Insulin-like growth factor binding protein levels have been demonstrated to be negatively correlated with birth weight (Verhaeghe et al., 1993). Another study reported that an increase in circulating relaxin concentrations was caused by hyperstimulation (Johnson et al., 1991), while high serum relaxin levels have been found to correlate to preterm birth (Weiss et al., 1993). Moreover, Buitendijk (2000) suggested that better perinatal outcome after transfer of cryopreserved embryos in human compared to regular IVF pregnancies might point to adverse effects of hyperstimulation. Multiple folliculogenesis in human or in vitro maturation of animal oocytes can result in the maturation of incompetent oocytes, which normally would not ovulate in the relevant oestrous cycle. Thus embryo technologies may use a pool of rescued oocytes with developmental defects, which would normally have died in vivo (Young \& Fairburn, 2000).

The in vitro period of assisted reproduction also seems to be relevant for the induction of the aberrant phenotype in livestock. Although ovarian hyperstimulation per se is sufficient to cause adverse outcomes in cattle, offspring produced after oocyte maturation in vitro followed by fertilization in vitro are affected more severely (Wagtendonk-de Leeuw et al., 2000). IVP calves differed significantly from calves born after hyperstimulation with regard to several features, including perinatal mortality risk, gestation length and birth weight. In addition, in vitro culturing of in vivo matured and fertilized ovine embryos can lead to LOS (Sinclair et al., 1997). Apparently, the in vitro period can negatively influence developing embryos.

In an attempt to mimic the oviduct environment, adjustments to the traditional culture conditions have been made by the application of culture media supplementation and the use of co-culture systems. Additional proteins and embryonic growth factors secreted by co-culture cells were thought to be beneficial to the fertilization process and development of the preimplantation embryo. Culture in serum-supplemented medium resulted in more bovine blastocysts than in medium without serum, but both treatments produced far fewer bovine blastocysts than did medium with co-culture (Hasler, 2000). In addition, medium with or without serum produced bovine embryos in a lower developmental stage, of lower quality and fewer cells than did medium with co-culture. However, calves born after transfer of co-cultured embryos showed a significantly higher birth weight, a prolonged gestation period and more calving problems than calves born following transfer of IVP embryos cultured in medium without co-culture (Wagtendonk-de Leeuw et al., 2000).

In vitro culture in the absence of serum and co-culture may also induce fetal abnormalities. Lane and Gardner (1994) demonstrated that early developing mouse embryos require amino acids for normal development. However, the amino acid waste product ammonium was shown to induce fetal retardation and exencephaly in a time- and concentration-dependent manner (Gardner \& Lane, 1993). By causing free oxygen radicals, culture under high oxygen percentages can damage the mitochondria (Menke \& McLaren, 1970). Kruip et al. (2000) proposed that oocytes with damaged mitochondria might have a perturbed oxidative phosphorylation and energy metabolism resulting in a perturbed feto-placental energy regulation, perturbed metabolism and overgrowth of the fetus.

Asynchronous embryo transfer and maternal progesterone administration are other factors known to be associated with LOS. Day 3 embryos placed in a day 6 uterus for a period of 3 days followed by transfer into another recipient synchronous with the embryo generated first trimester ovine fetuses with an increased weight (Young et al., 1996). Abnormal growth of these ovine fetuses is suggested to be the result of asynchrony between the embryo and the uterine endometrium. Maternal progesterone administration 
during the first days of pregnancy also enhances embryonic development and increases fetal weight in sheep (Kleeman et al., 1994).

Although extrapolating data from animal to human and vice versa is hampered due to differences in physiology and reproductive technologies, sufficient evidence has been provided to conclude that assisted reproduction can affect fetal development.

\section{Genomic Imprinting}

Abnormalities and aberrant fetal growth observed after assisted reproduction in human as well as in animal seem to occur after exposure of gametes or preimplantation embryo to an unusual environment. Therefore, the programming of abnormal development must occur prior to follicle aspiration in the ovary or during the culture period of 120 hours. During gametogenesis and early embryonic development expression of imprinted genes (i.e. genes which are only expressed from one of the parental alleles) is regulated by epigenetic modifications (Young \& Fairburn, 2000). There is substantial evidence that modification of some germ line imprints in preimplantation embryos can be affected by assisted reproduction. Early embryonic epigenetic alterations in imprinted genes arisen from derivation or culture of mouse embryonic stem (ES) cells persist to later developmental stages and are associated with aberrant gene expression in ES cell derived fetuses (Dean et al., 1998). Aberrant phenotypes observed after assisted reproduction are likely to result from any similar genomic alterations, which are not corrected after their induction in early development. Of particularly significance to embryo technologies are those imprinted genes with products known to be associated with developmental abnormalities.

Insulin-like growth factor II (IGF2) plays a key role in fetal growth and development, influencing fetal cell division, differentiation and possibly metabolic regulation (reviewed by Latham, 1999). Gene-targeting methods in the mouse demonstrated that one copy of the Igf2 gene was silenced by genomic imprinting. Knockout studies demonstrated that mice inheriting a paternal null allele are about $60 \%$ of normal body weight, whereas inheritance of the maternal null allele has no effect on growth (De Chiara et al., 1990). Loss of imprinting of Igf2, resulting in biallelic expression of Igf2, is the most common molecular defect found in the human Beckwith-Wiedemann syndrome (Reik \& Maher, 1997). The most striking features of this syndrome are an increase in birth weight, organ overgrowth, polyhydramnios and childhood tumors. Similar abnormalities have been observed after overexpression of Igf2 in the mouse. The H19 gene and/or its promoter are suggested to be necessary for imprinted regulation of the Igf2 gene (Leighton et al., 1995). Mice with disturbed maternal H19 alleles express no H19, consequently express Igf2 from both alleles and produce increased levels of tissue IGF2. Another imprinted gene known to be associated with the induction of overgrowth is Igf2r. Disruption of the maternally expressed Igf $2 \mathrm{r}$ allele in the mouse causes developmental abnormalities, including increased birth weight, organ overgrowth, polyhydramnios and reluctance to suckle (Eggenschwiler et al., 1997). Apparently, persisting developmental abnormalities can result from a disturbed regulation of imprinted gene expression during early embryonic development.

Involvement of the imprinted genes Igf2, H19 and Igf2r in the induction of LOS has been suggested, since disturbance of these genes was found to cause similar phenotypes as those reported for IVP livestock. Although unravelling the role of any imprinted genes in LOS has been hampered, substantial evidence has recently been provided by Young et al. (2001) by demonstrating that epigenetic change in Igf $2 \mathrm{r}$ was associated with fetal overgrowth after sheep culture. The implication of other imprinted genes in causing fetal overgrowth is still under examination. Whether similar genes or pathways are involved in the induction of abnormal development in human assisted reproduction also remains to be established.

\section{Long Term Consequences}

Little is known about long term consequences of growth abnormalities caused by assisted reproduction on postnatal human and animal health. Weight of sheep and calves aged $\geq 1$ year with an increased birth weight is similar to those of sheep and calves with a normal birth weight (McEvoy et al., 1998). However, abnormal large hearts have been found in IVP calves aged 1 year and oversized at birth (McEvoy et al., 1998). Effects of allometric organ size and reported perinatal abnormalities in IVP offspring on postnatal health should be extensively investigated.

Since the introduction of IVF was not accompanied by formal evaluation of possible adverse effects on health and due to the relatively short time that elapsed since the first IVF baby was born in 1978, current knowledge on postnatal health of IVF children is rather limited. Cederblad et al. (1996) demonstrated that the cognitive, behavioural, psychosocial and physical development of 2-year-old children born after IVF was normal.

Epidemiological studies in human, using data from individuals born after natural conception, have found associations between prenatal events and chronic disease in later life. Low birth weight, thinness and short body length at birth are now known to be associated with increased rates of cardiovascular disease and diabetes type II in adult life (Barker, 1995). Several European studies have gained support for the fetal origin hypothesis (Law et al., 1996; Leon et al., 1996). The Dutch famine has provided the opportunity to elucidate the effects of intrauterine growth restriction in human. Body size at birth, especially in babies exposed to famine during late gestation, has been found to be negatively affected (Smith, 1947). In addition, prenatal exposure to famine, especially during mid and late gestation, is associated with decreased glucose tolerance in adult life (Ravelli et al., 1998).

As discussed previously, the IVF procedure differs from the natural conception at various levels, leading to the suggestion that conditions during the IVF procedure might interfere with fetal growth and development and thereby influencing the programming of organs and their function. Further research will be necessary to investigate whether, just as intrauterine growth retarded individuals, IVF children are at increased risk for chronic disease, such as diabetes type II 
and cardiovascular disease, in later life. Nowadays, no indications are present which suggests long term effects of IVF on offspring with regard to other health aspects including behaviour, fertility or oncology.

Other forms of assisted reproduction have been developed, including nuclear transfer (cloning) in livestock. Developmental abnormalities in cattle generated after the fusion of a nuclear karyoplast with an enucleated oocyte are more severe and are observed more often as compared to those reported for IVP (Wilson et al., 1995; Garry et al., 1996). In addition, further evaluation of nuclear transfer is hindered due to the relatively low number of cloned ruminants that survive to term. These findings could be interpreted as a warning to human assisted reproduction. They seem to indicate that the larger the difference between natural conception and the assisted reproduction technology used, the greater will be the adverse effects on the health of generated offspring. Intracytoplasmic sperm injection (ICSI), a procedure in which a single sperm is injected directly into an oocyte, is currently the most manipulative assisted reproduction technology used in human. Fortunately, results of studies investigating the development of ICSI children up to two years of age are quite reassuring (Bonduelle et al., 1998; Bonduelle et al., 1999). However, until further and more extensive follow-up studies are conducted, it remains unclear whether any of the currently used assisted reproduction technologies both in human and livestock affect health in later life.

\section{References}

Barker, D. J. P. (1995). Fetal origins of coronary heart disease. British Medcial Journal, 311, 171-174.

Bonduelle, M., Camus, M., De Vos, A., Staessen, C., Tournaye, H., Van Assche, E., Verheyen, G., Devroey, P., Liebaers, I., \& Van Steirteghem, A. (1999). Seven years of intracytoplasmic sperm injection and follow-up of 1987 subsequent children. Human Reproduction, Suppl. 1, 243-264.

Bonduelle, M., Joris, H., Hofmans, K., Liebaers, I., \& Van Steirteghem, A. (1998). Mental development of 201 ICSI children at 2 years of age. Lancet, 351, 1553.

Buitendijk, S. E. (1999). Children after in vitro fertilization. An overview of the literature. International Journal of Technology Assessment in Health Care, 15, 52-65.

Buitendijk, S. E. (2000). IVF pregnancies: Outcome and follow-up. Unpublished doctoral dissertation, Universiteit Leiden, the Netherlands.

Cederblad, M., Friberg, B., Ploman, F., Sjöberg, N. O., Stjernqvist, K., \& Zackrisson, E. (1996). Intelligence and behaviour in children born after in-vitro fertilization treatment. Human Reproduction, 11, 2052-2057.

De Chiara, T. M., Efstradiatis, A., \& Robertson, E. J. (1990). A growth-deficiency phenotype in heterozygous mice carrying an insulin-like growth factor II gene disrupted by targeting. Nature, 345, 78-80.

De Mouzon, J., \& Lancaster, P. (1997). World collaborative report on in vitro fertilization, preliminary data for 1995 . Journal of Assisted Reproduction and Genetics, 14S, 251-265.

Dean, W., Bowden, L., Aitchison, A., Klose, J., Moore, T., Meneses, J. J., Reik, W., \& Feil, R. (1998). Altered imprinted gene methylation and expression in completely ES cell-derived mouse fetuses: Association with aberrant phenotypes. Development, 125, 2273-2282.

Eggenschwiler, J., Ludwig, T., Fisher, P., Leighton, P. A., Tilghman, S. M., \& Efstratiadis, A. (1997). Mouse mutant embryos overexpressing IGF-II exhibit phenotypic features of the Beckwith-Wiedemann and Simpson-Golabi-Behmel syndromes. Genes \& Development, 11, 3128-3142.

Ertzeid, G., \& Storeng, R. (2001). The impact of ovarian stimulation on implantation and fetal development in mice. Human Reproduction, 16, 221-225.

Gardner, D. K., \& Lane, M. (1993). Amino acids and ammonium regulate mouse embryo development in culture. Biology of Reproduction, 48, 377-385.

Garry, F. B., Adams, R., McCann, J. P., \& Odde, K. G. (1996). Postnatal characteristics of calves produced by nuclear transfer cloning. Theriogenology, 45, 141-152.

Hasler, J. F. (2000). In vitro culture of bovine embryos in Menezo's B2 medium with or without coculture and serum: The normalcy of pregnancies and calves resulting from transferred embryos. Animal Reproduction Science, 60-61, 81-91.

Hasler, J. F., Henderson, W. B., Hurtgen, P. J., Jin, Z. Q., McCauley, A. D., Mower, S. A., Neely, L., Shuey, S., Stokes, J. E., \& Trimmer, S. A. (1995). Production, freezing and transfer of bovine IVF embryos and subsequent calving results. Theriogenology, 43, 141-152.

Holm, P., Walker, S. K., \& Seamark, R. F. (1996). Embryo viability, duration of gestation and birth weight in sheep after transfer of in vitro matured and in vitro fertilized zygotes cultured in vitro or in vivo. Journal of Reproduction and Fertility, 107, $175-181$.

Johnson, M. R., Irvine, R., Hills, F., Bolton, V. N., Abbas, A. A., Brooks, A. A., Allman, A. C., Chard, T., \& Nicolaides, K. H. (1995). Superovulation, IGFBP-1 and birth weight. European Journal of Obstetrics, Gynecology and Reproductive Biology, 59, 193-195.

Johnson, M. R., Okokon, E., Collins, W. P., Sharma, V., \& Lightman, S. L. (1991). The effect of human chorionic gonadotropin and pregnancy on the circulating level of relaxin. The Journal of Clinical Endocrinology and Metabolism, 72, 1042-1047.

Kleeman, D. O., Walker, S. K., \& Seamark, R. F. (1994). Enhanced fetal growth in sheep administered progesterone during the first three days of pregnancy. Journal of Reproduction and Fertility, 102, 411-417.

Koudstaal, J., Braat, D. D., Bruinse, H. W., Naaktgeboren, N., Vermeiden, J. P., \& Visser, G. H. (2000). Obstetric outcome of singleton pregnancies after IVF: A matched control study in four Dutch university hospitals. Human Reproduction, 15, 1819-1825.

Koudstaal, J., Bruinse, H. W., Helmerhorst, F. M., Vermeiden, J. P., Willemsen, W. N., \& Visser, G. H. (2000). Obstetric outcome of twin pregnancies after in-vitro fertilization: A matched control study in four Dutch university hospitals. Human Reproduction, 15, 935-940.

Koudstaal, J. (1999). Course and outcome of Dutch IVF pregnancies. Unpublished doctoral dissertation, Universiteit Utrecht, the Netherlands. 
Kruip, T. A. M., Bevers, M. M., \& Kemp, B. (2000). Environment of oocyte and embryo determines health of IVP offspring. Theriogenology, 53, 611-618.

Kruip, T. A. M., \& den Daas, J. H. G. (1997). In vitro produced and cloned embryos: Effects on pregnancy, parturition and offspring. Theriogenology, 47, 43-52.

Lambalk, C. B., \& Hooff, M. (2001). Natural versus induced twinning and pregnancy outcome: A Dutch nationwide survey of primiparous dizygotic twin deliveries. Fertility and Sterility, 75, 731-736.

Lane, M., \& Gardner, D. K. (1997). Differential regulation of mouse embryo development and viability by amino acids. Journal of Reproductive Fertility, 109, 153-164.

Lane, M., \& Gardner, D. K. (1994). Increase in postimplantation development of cultured mouse embryos by amino acids and induction of fetal retardation and exencephaly by ammonium ions. Journal of Reproductive Fertility, 120, 305-312.

Latham, K. E. (1999). Epigenetic modification and imprinting of the mammalian genome during development. Current Topics in Developmental Biology, 43, 1-49.

Law, C. M., Martyn, C. N., Fall, C. H., \& Osmond, C. (1996). Raised adult blood pressure linked to failure to achieve growth potential in utero. British Medical Journal, 312, 1479.

Leighton, P. A., Ingram, R. S., Eggenschwiler, J., Efstratiadis, A., \& Tilghman, S. M. (1995). Disruption of imprinting caused by deletion of the H19 gene region in mice. Nature, 375, 34-39.

Leon, D. A., Koupilova, I., Lithell, H. O., Berglund, L., Mohsen, R., Vagero, D., Lithell, U-B. \& McKeigue, P. M. (1996). Failure to realise growth potential in utero and adult obesity in relation to blood pressure in 50 year old Swedish men. British Medical Journal, 312, 401-406.

Lohuis, M. M. (1995). Potential benefits of bovine embryo-manipulation technologies to genetic improvement programs. Theriogenology, 43, 51-60.

McEvoy, T. G., Sinclair, K. D., Broadbent, P. J., Goodhand, K. L., \& Robinson, J. J. (1998). Post-natal growth and development of Simmental calves derived from in vivo or in vitro embryos. Reproduction, Fertility, and Development, 10, 459-64.

Menke, T. A., \& McLaren, A. (1970). Mouse blastocysts grown in vivo and in vitro: Carbon dioxide production and trophoblast outgrowth. Journal of Reproduction and Fertility, 23, 117-127.

Ravelli, A. C., van der Meulen, J. H., Michels, R. P., Osmond, C., Barker, D. J., Hales, C. N., \& Bleker, O. P. (1998). Glucose tolerance in adults after prenatal exposure to famine. Lancet, 351, 173-177.

Reik, W., \& Maher, E. R. (1997). Imprinting in clusters: Lessons from Beckwith-Wiedemann syndrome. Trends in Genetics, 13, 330-334.
Sinclair, K. D., Maxfield, E. K., Robinson, J. J., Maltin, C. A., McEvoy, T. G., Dunn, L. D., Young, L. E., \& Broadbent, P. J. (1997). Culture of sheep zygotes can alter fetal growth and development. Theriogenology, 47, 380.

Sinclair, K. D., McEvoy, T. G., Carolan, C., Maxfield, E. K., Maltin, C. A., Young, L. E., Wilmut, I., Robinson, J. J., \& Broadbent, P. J. (1998). Conceptus growth and development following in vitro culture of ovine embryos in media supplemented with bovine sera. Theriogenology, 49, 218.

Smith, C. (1947). The effects of wartime starvation in Holland upon pregnancy and its product. American Journal of Obstetrics and Gynecology, 53, 599-608.

Verhaeghe, J., Van Bree, R., Van Herck, E., Laureys, J., Bouillon, R., \& Van Assche, F. A. (1993). C-peptide, insulin-like growth factors I and II, and insulin-like growth factor binding protein1 in umbilical cord serum: Correlations with birth weight. American Journal of Obstetrics and Gynecology, 169, 89-97.

Wagtendonk-de Leeuw van, A. M., Aerts, B. J. G., \& den Daas, J. H. G. (1998). Abnormal offspring following in vitro production of bovine preimplantation embryos: A field study. Theriogenology, 49, 883-894.

Wagtendonk-de Leeuw van, A. M., Mullaart, E., de Roos, A. P. W., Merton, J. S., den Daas, J. H. G., Kemp, B., \& de Ruigh, L. (2000). Effects of different reproduction techniques: AI, MOET or IVP, on health and welfare of bovine offspring. Theriogenology, 53, 575-597.

Weiss, G., Goldsmith, L. T., Sachdev, R., von Hagen, S., \& Lederer, K. (1993). Elevated first-trimester serum relaxin concentrations in pregnant women following ovarian stimulation predict prematurity risk and preterm delivery. Obstetrics and Gynecology, 82, 821-828.

Wilson, J. M., Williams, J. D., Bondioli, K. R., Looney, C. R., Westhusin, M. E., \& McCalla, D. F. (1995). Comparison of birth weight and growth characteristics of bovine calves produced by nuclear transfer (cloning), embryo transfer and natural mating. Animal Reproduction Science, 38, 73-83.

Young, L. E., Butterwith, S. C., \& Wilmut, I. (1996). Increased ovine fetal weight following transient asynchronous embryo transfer is not associated with increased placental weight at day 21 of gestation. Theriogenology, 45, 231.

Young, L. E., \& Fairburn, H. R. (2000). Improving the safety of embryo technologies: possible role of genomic imprinting. Theriogenology, 53, 627-648.

Young, L. E., Fernandes, K., McEvoy, T. G., Butterwith, S. C., Gutierrez, C. G., Carolan, C., Broadbent, P. J., Robinson, J. J., Wilmut, I., \& Sinclair, K. D. (2001). Epigenetic change in IGF2R is associated with fetal overgrowth after sheep embryo culture. Nature Genetics, 27, 153-154.

Young, L. E., Sinclair, K. D., \& Wilmut, I. (1998). Large offspring syndrome in cattle and sheep. Reviews of Reproduction, 3, 155-163. 